\title{
New associate Editor Prof. Dr. med. Christina Fotopoulou
}

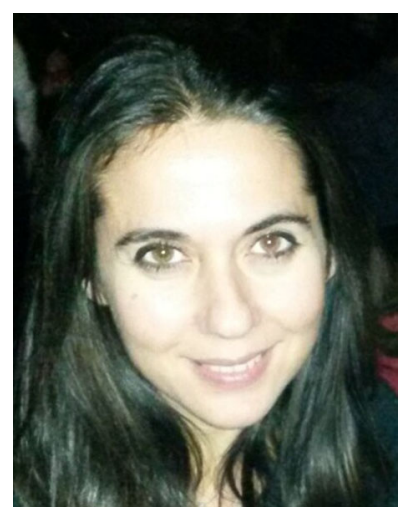

Christina Fotopoulou trained in 2002 in obstetrics and gynecology and subspecialized in gynecological oncology at the Charité University Hospital of Berlin in the surgical and systemic treatment of women with advanced gynecological malignancies and completed her Ph.D. thesis about "Current Aspects in the Operative Treatment of Epithelial Ovarian Cancer".

She is since 2013 a Consultant gynecological Oncologist in the Imperial College London Healthcare Trust in Queen Charlottes Hospital in London, and is a principal investigator of the Ovarian Cancer Action Research Centre, UK, leading the bioengineering group of the center.

She has been the Vice Director of the Clinic for Gynecology at the Charité in Berlin, one of the largest references and accredited centers for gynecological cancer in Germany, as well the Principal Coordinator of the European Competence Center for Ovarian Cancer, which was created in 2007 in Berlin.

Her principal area of clinical practice is in exenterative procedures for advanced forms of pelvic malignancies and in the cytoreductive debulking of primary or relapsed ovarian cancer and the investigation of predictive and prognostic biomarkers of surgical outcome.

She is a member of the German AGO-Ovarian Cancer Steering Group and is the surgical lead in the European Network for Translational Research in Ovarian Cancer (EUTROC) in the working package "Predicting surgical outcome".

She is on the editorial board of three journals and reviewer in numerous international gynecological and oncological journals and is member of various international oncological committees, including ASCO, ESGO, IGCS, ESMO, ENGOT, AGO and NOGGO. 